... Bertrand Kiefer: Arzt, Priester, Ethiker - und Chefredaktor der «Revue Médicale Suisse»

\title{
«Die Reflexion ist meine Leidenschaft»
}

\section{Daniel Lüthi}

Text und Bilder
Es ist wohl eher selten, dass es bei einem Arzt nach Druckerschwärze riecht. Bei Bertrand Kiefer ist es so, und auch sonst ist bei ihm vieles anders als bei anderen. So hat er viele Berufe, die zu einer Berufung geworden sind.

\section{Medizin - eine Leiche?}

$\mathrm{Zu}$ seinem Büro gelangen wir via Papierlager und Druckerei. Und dort sprechen wir gleich über ein Buch, das hier nicht nur verlegt und gedruckt, sondern auch geschrieben wurde: «Le cadavre de la médecine bouge encore» - die Medizin als Leiche, die sich noch ein wenig regt. Eine Kollektion von rund 170 Kolumnen, die Kiefer im Laufe der letzten zehn Jahre für seine Zeitschrift, die «Revue Médicale Suisse», geschrieben hat; eine Sammlung von tiefschürfenden Gedanken zu grossen Themen. Für den Beginn eines neuen Jahres genau das Richtige. Viel Philosophisches, aber auch viel Kritisches findet sich darin. Bisweilen wirkt das Ganze reichlich pessimistisch.

Am Anfang kommt gleich der Blick auf den Schluss: Das Buch beginnt mit der Frage «Fin d'humanité?» Nichts Geringeres als das Ende der

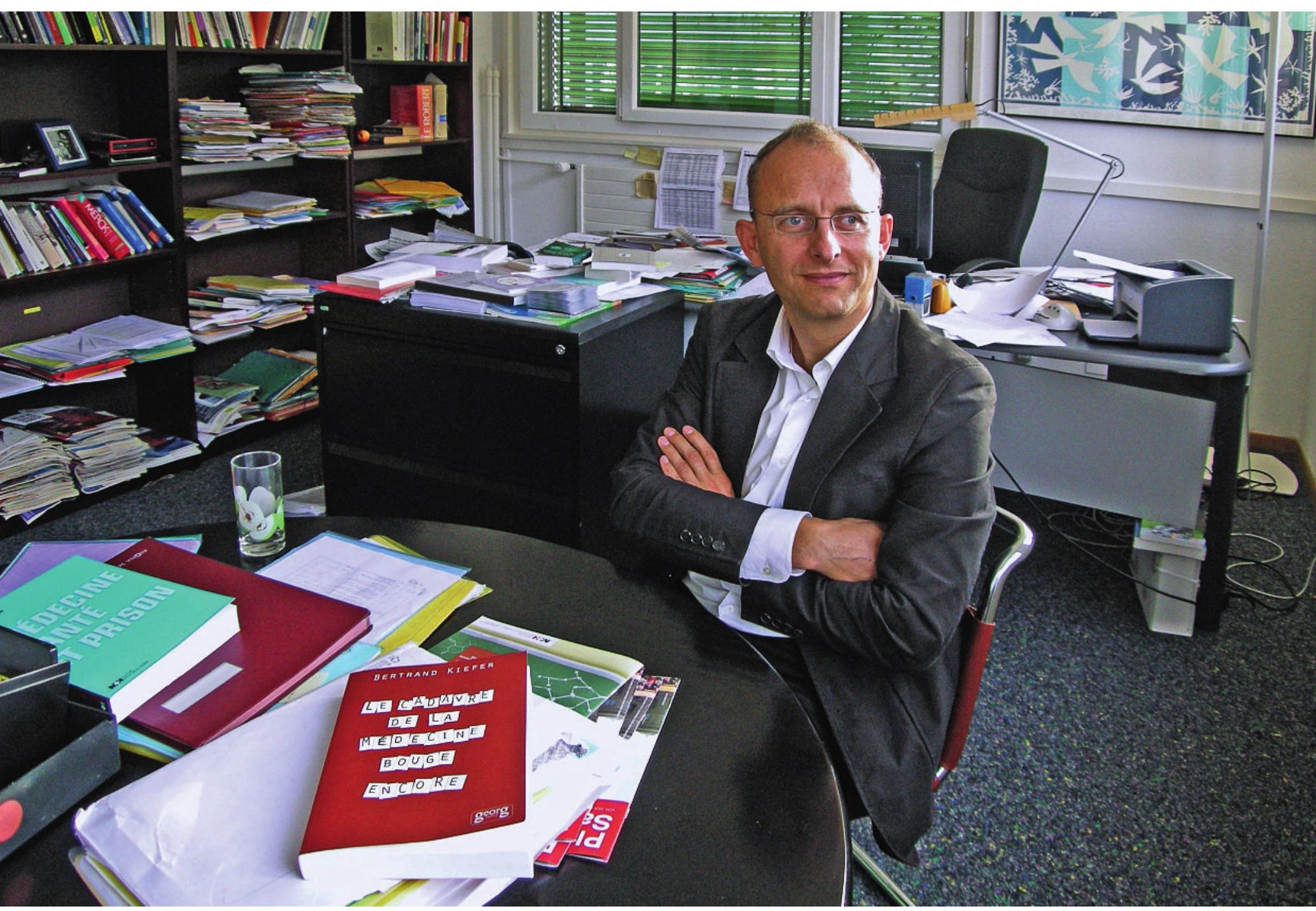


Menschlichkeit, und damit vielleicht auch das Ende der Menschheit, stehen zur Diskussion. «Nein, ich bin kein Pessimist», wehrt sich Kiefer, «auch nicht a priori ein Optimist. Ich versuche, die Dinge so zu sehen, wie sie sind, bin also eher ein Realist.» Bezogen auf die Medizin heisst das für ihn: «Alle Kulturen sind sterblich, deshalb kann auch die Medizin verschwinden.» Gemeint ist die Essenz der Medizin - eben: die Menschlichkeit. Immer mehr werde die Medizin zur «Heilungs-Maschinerie», immer mehr habe sie sich dem Diktat der Wirtschaft zu beugen, die eine humanitäre Vision verunmögliche. Effizienz statt Empathie. Zahlen statt Zuneigung. Und die Medizin als blosses Konsumgut, als ein Business wie andere auch: «Technologien, virtuelle Welt und Management drängen die Person zunehmend in den Hintergrund», sagt Kiefer, dabei gehe es für eine Ärztin oder einen Arzt doch darum, «einem Menschen zu helfen, sich selber sein zu können.» Ein Beispiel hilft, zu verstehen: «Eine alte inkontinente Frau, die Diabetes hat und Mühe beim Gehen - Ziel ihres Hausarztes kann doch nicht sein, sie mit jedem erdenklichen Aufwand von all dem zu heilen?» Einmal mehr stellt Betrand Kiefer eine Frage, statt gleich eine Antwort zu präsentieren, auch das ist typisch für ihn.

\section{«Technologien, virtuelle Welt und Management drängen die Person zunehmend in den Hintergrund.»}

\section{Vom Priester zum Journalisten}

Bertrand Kiefer ist, wie er selber sagt, «ein Arzt, der eigentlich nie als Arzt gearbeitet hat». Er schreibt, philosophiert und kritisiert, legt den Finger auf wunde Stellen der Gesellschaft und versucht, die Dinge immer wieder aus der Distanz zu betrachten. Am Lack kratzen, recherchieren, ausgraben und aufdecken, hinter die Kulissen schauen: Als Journalist fühlt er sich wohl. Bloss: «Leider fehlt in der Schweiz zunehmend das Geld für solche Arbeit - und das ist ein Problem für die Demokratie.» Jetzt gerade scheint ein Politiker zu sprechen, das jedoch will Kiefer explizit nicht sein. Eher identifiziert er sich mit Philosophen wie Peter Sloterdijk, Michel Foucault, Jean Baudrillard oder Georges Canguilhem. Letzterer hat sich beispielsweise Gedanken gemacht über die fliessenden Grenzen zwischen dem Normalen und dem Krankhaften. Bei solchen Themen gerät Kiefer geradezu ins Schwärmen: «Ich liebe es, nachzudenken die Reflexion ist meine Leidenschaft.»

Und wie steht es mit der Religion, immerhin war dieser Arzt und Journalist auch einmal katholischer Priester? Das scheint eine wunde Stelle zu sein. Angesprochen auf die Gründe, warum er Rom und die Kirche damals verlassen hat, spricht Bertrand Kiefer, offensichtlich geniert und bewusst abstrakt, von

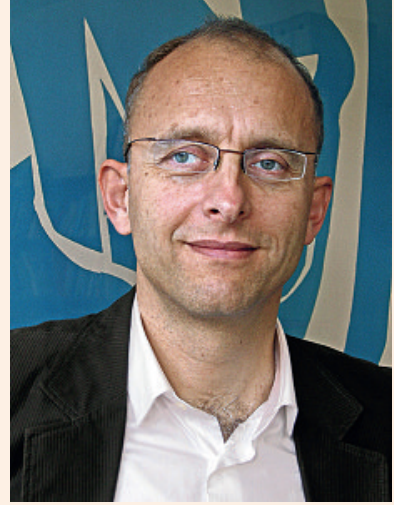

\section{Bertrand Kiefer}

Dr. med. Bertrand Kiefer wurde 1955 in Genf geboren, als Sohn eines Arztes. Schulen und Medizinstudium absolvierte er ebenfalls in Genf, 1981 machte er hier auch sein Staatsexamen. Ein Jahr lang forschte er anschliessend im Gebiet der Infektionskrankheiten - und ging dann nach Freiburg, wo er Theologie studierte. 1988 empfing Bertrand Kiefer die Priesterweihe. In Rom - zum Teil im Vatikan - arbeitete er dann drei Jahre lang an einer Doktorarbeit im Themenbereich «Gentechnik». Verschiedene Gründe veranlassten ihn dann, Rom und den kirchlichen Weg zu verlassen. Zurück in der Schweiz arbeitete er zwischen 1991 und 1993 je zur Hälfte als Journalist (beim «Journal de Genève») und als Psychiater in einem Ambulatorium des Universitätsspitals. 1993 wurde er Chefredaktor der Wochenzeitschrift «Médecine \& Hygiène», die 2005 mit der «Revue Médicale de la Suisse Romande» fusionierte und zur «Revue Médicale Suisse» wurde. Seit dem Jahre 2000 ist Kiefer zusätzlich Direktor von Verlag und Druckerei bei «Médecine \& Hygiène».

Bertrand Kiefer ist mit einer Fernsehjournalistin verheiratet. Mit ihr und deren Sohn lebt er in der Nähe von Genf. 
einer schwierigen persönlichen Situation in jener Zeit, von Dogmen und doktrinären Phänomenen, die er nicht mehr akzeptieren wollte - und von einer Frau, in die er sich verliebt hatte.

\section{Was bringt 2011?}

Schauen wir nach vorne, statt zurück - blicken wir auf das neue Jahr, 2011. «Managed Care und Einheitskrankenkasse werden Themen sein.» Kiefer befürwortet eine bessere Vernetzung insbesondere zwischen Spitälern und ambulanter Medizin, und er ist auch dafür, dass die Krankenkassen das Soziale und das Private trennen.

Weiter: «Über die DRGs, die diagnosebezogenen Fallpauschalen werden wir sprechen. Sie werden unser Gesundheitssystem so richtig durchschütteln. Und für besonders verletzliche Gruppen, chronisch Kranke oder Polymorbide zum Beispiel, werden sie zum Problem. Denn die Leute sollen ja so wenig wie möglich kosten. Wenn also ein Patient zum Beispiel sagt: ‘ch habe Angst), muss die Pflegende antworten: 〈Und ich habe keine Zeit.〉 Von den Erfahrungen in Deutschland haben wir gelernt, dass dieser zunehmende Druck gerade bei den Pflegenden vermehrt zu Burnouts führt.» Er sei nicht à priori gegen das neue System, präzisiert Kiefer. Einmal mehr aber plädiert er entschieden dafür, genauer hinzuschauen.

\section{Wirtschaftlichkeit ja, aber nicht um jeden Preis.}

Und was bringt das neue Jahr ihm selber? Interessante Diskussionen in der Nationalen Ethikkommission, zu grossen Fragen wie Präimplantations-Diagnostik oder Suizidbeihilfe. Erneut also, auf hohem Niveau, die Auseinandersetzung mit Leben und Sterben, Geburt und Tod.
Und ganz persönlich? Welches Verhältnis hat Bertrand Kiefer zu seinem eigenen Tod? Wie gewohnt hört er sich die Frage zuerst aufmerksam an, überlegt dann, lässt den Blick in die Ferne schweifen und zurück zum Gegenüber, lächelt - und antwortet: «Ein seltsames Mysterium. Der Tod ist überall in der Natur. Ja: Er beschäftigt mich. Früher wünschte man sich ja, dass man den Tod würde kommen sehen, um sich darauf vorbereiten zu können. Heute ist ein 'guter Tod eher ein plötzlicher, unerwarteter. So gesehen bin ich ein moderner Mensch: Ich möchte leben und dann unvermittelt und schnell sterben.» Genussvoll leben heisst für Kiefer zum Beispiel: aufgehoben sein in der Familie und bei Freunden. Diskussionsrunden. Oder wandern und Ski fahren in den Bergen, wie über die Festtage jeweils im Wallis.

Natürlich wird Bertrand Kiefer dieses Jahr auch viel Alltägliches, Geschäftliches beschäftigen, schliesslich ist er ja nicht zuletzt Direktor eines Unternehmens mit 35 Beschäftigten: Personalfragen in Verlag und Druckerei stehen an - und die Kreation eines grossen Internetportals für die Romandie zum Thema Gesundheit. «Was muss ich tun, wenn ...» oder «Was sind die Anzeichen eines Notfalls?» sind Fragen, die hier einem breiten Publikum beantwortet werden sollen. Eine weitreichende Vernetzung mit Spitälern, kantonalen Ärztegesellschaften oder spezialisierten Ligen sei wichtig bei diesem Projekt, sagt Kiefer. Und: «Da ist viel unternehmerisches Risiko drin, gerade deshalb, weil die kommerzielle Idee nicht im Vordergrund steht.»

Einmal mehr: Wirtschaftlichkeit ja, aber nicht um jeden Preis.

Und, noch einmal: Die vielen Berufe des Bertrand Kiefer scheinen zu einer Berufung verschmolzen zu sein. Der Arzt, Priester, Ethiker, Philosoph, Journalist, Chefredaktor und Verlagsdirektor ist vor allem eines: ein Mensch, der gerne nachdenkt.

\section{Die nächste «Begegnung mit ...»}

Am Ende jeden Monats stellt die Schweizerische Ärztezeitung eine Persönlichkeit vor, die sich im Gesundheitswesen engagiert. Im Februar schildert Daniel Lüthi seine Begegnung mit Lutgard Werlen, Allgemeinmedizinerin, einzige Ärztin im Lötschental und Bergsportlerin. 